\title{
Circulating microRNA-194 regulates human melanoma cells via PI3K/AKT/FoxO3a and p53/p21 signaling pathway
}

\author{
MING BAI, MINGZI ZHANG, FEI LONG, NANZE YU, ANG ZENG and RU ZHAO
}

Department of Plastic and Reconstructive Surgery, Peking Union Medical College Hospital, Chinese Academy of Medical Science and Peking Union Medical College, Beijing 100730, P.R. China

Received September 26, 2016; Accepted March 7, 2017

DOI: $10.3892 / o r .2017 .5537$

\begin{abstract}
In the present study, we analyzed the role of microRNA-194 circulating regulated human melanoma cell growth. We found that microRNA-194 expression was markedly suppressed in human melanoma patients, compared with negative control group. Next, disease-free survival (DFS) and overall survival (OS) of high expression in human melanoma patients was higher than those of low expression in human melanoma patients. MicroRNA-194 overexpression inhibited cell proliferation, induced apoptosis, increased caspase-3/-9 activities and promoted Bax/Bcl-2 of human melanoma cells. Furthermore, microRNA-194 overexpression also suppressed PI3K/AKT/FoxO3a signaling pathway and induced p53/p21 signaling pathway. PI3K inhibitor, suppressed PI3K, phosphorylation-AKT, FoxO3a protein expression and increased the effects of microRNA-194 overexpression on cell growth, apoptosis, caspase-3/-9 activities and $\mathrm{Bax} / \mathrm{Bcl}-2$ protein expression of human melanoma cells through the induction of p53/p21 signaling pathway. Taken together, these data indicate that circulating microRNA-194 regulated human melanoma cells via PI3K/AKT/FoxO3a and p53/p21 signaling pathway.
\end{abstract}

\section{Introduction}

Malignant melanoma (MM) is a highly malignant tumor that mostly strikes adults aged more than 30 years, and the elderly, and it has high mortality. The morbidity of MM has increased gradually in recent years and shows a younger trend $(1,2)$. It accounts for $\sim 2 \%$ of malignancies in terms of morbidity and ranks the third of skin malignancies (6.8-20\%); the disease can occur in any part of the skin, which is dominant by the sites that are vulnerable to friction in the four extremities, and its

Correspondence to: $\mathrm{Dr} \mathrm{Ru}$ Zhao, Department of Plastic and Reconstructive Surgery, Peking Union Medical College Hospital, Chinese Academy of Medical Science and Peking Union Medical College, No. 1 Shuaifuyuan, Dongcheng, Beijing 100730, P.R. China E-mail: zhaoruruzhao6114@163.com

Key words: microRNA-194, melanoma cells, PI3K/AKT/FoxO3a, p53/p21 characteristics are early metastasis, strong invasiveness and poor prognosis (3). The treatment at present is mainly surgical treatments, and the subsequent biotherapy, radiotherapy and chemotherapy is not clinically effective, thus, it has poor prognosis and high mortality (4).

Recently, with the continuous development of molecular biology, great progresses have been achieved in research on genetics and molecular biology, such as the genesis, development and metastasis of MM, which has greatly promoted the clinical treatment and basic research on MM (5). However, there is little research on the effect of miRNA on MM as well as its mechanism of action (5).

MicroRNA is a type of non-coding small RNA consisting of 18-25 nucleotides that was newly discovered to be closely associated with MM (6). MicroRNA plays an important regulatory role in normal human physiological function, which can regulate the target gene and influence the expression of its protein; while under some conditions, the increased or decreased expression of the key protein will lead to cellular functional changes, or even the genesis of tumor (7). The expression profiles of microRNA are greatly different between tumor cells and normal tissue cells (7). A majority of microRNAs are associated with high expression, low expression or expression deletion in diverse tumor cells, the abnormal expression of which is one of the important molecular mechanisms of participating tumor cell proliferation and growth (8).

Akt is the key downstream effector molecule of PI3K, which can promote tumor growth and invasion through promoting angiogenesis and migration-associated procedures after being phosphorylated and activated. PTEN can dephosphorylate phosphatidylinositol 3,4,5-trisphosphate (PIP3), and thus, weaken the PI3K-derived activation signal and indirectly inhibit the activation of Akt (9). The low expression of PTEN results in its disability to effectively inhibit the abnormal activation of the PDK/Akt pathway, and thus leads to tumor migration, which is associated with the highest occurrence rate in prostate, endometrial cancer, glioma and MM (10). As is reported, PTEN gene changes can be seen in $30-50 \%$ of MM cell lines and 5-20\% of MM cells $(9,11)$. The activated PI3K/Akt may further activate its downstream molecule mTOR through the TSC1/2 complex, and the activated mTOR can activate its two downstream molecules subsequently, which are the translation inhibitory 
molecule eIF-4E binding protein 1 (4E-BP1) and ribosomal protein p70S6K $(10,12)$. The activation of p70S6K can promote the filament reconstruction of the actin and promote cell movement $(12,13)$.

$\mathrm{p} 21$, is a CDK kinase inhibitor belonging to the Cip/Kip CDK kinase inhibitor family, and has multiple activities in cancer (14). p21 has two non-overlapped domains, which are the PCNA binding domain locating in the carboxyl-terminal and the CDK-cyclin inhibiting domain in the amino-terminal. p21 binds with the cyclin through the Cyl motif in the $\mathrm{N}$-terminal, and it can also bind with CDK subunit through the CDK binding locus locating in the N-terminal which separates from the $\mathrm{Cy} 1$ motif. Under certain specific conditions, p21 can promote the kinase activities of CDK4 and CDK6, thus, promoting the cells to pass stage G1; p21 can inhibit the activity of CDK2 and thus, inhibit the phosphorylation of the CDK2-dependent RB protein, and the unphosphorylated $\mathrm{RB}$ can bind with the transcription factor E2F1 and thus, inhibit its transcriptional activity; therefore, p21 inhibits CDK2 and eventually inhibits the transcription of the gene necessary for the E2F1-dependent G1/S transformation, leading to stage G1/S arrest $(2,15)$. Targeted knockout CDK2 demonstrates that CDK2 is not necessary for the inhibitory effect of p21 on cell cycle; and p21 can also inhibit the activity of CDK1 kinase, thus, rendering the arrest of cell cycle in the G2/M stage $(2,16)$. The present study addressed the role of microRNA-194 circulation-regulated human melanoma cell growth.

\section{Materials and methods}

Patients and tissue samples. MM samples and matched adjacent normal tissues were obtained from patients in the Department of Plastic and Reconstructive Surgery, Peking Union Medical College Hospital, Chinese Academy of Medical Science and Peking Union Medical College. The samples were stored at liquid nitrogen after the collection. The study was approved by the Ethics Committee of the Department of Plastic and Reconstructive Surgery, Peking Union Medical College Hospital, Chinese Academy of Medical Science and Peking Union Medical College (Table I). Disease-free survival (DFS) and overall survival (OS) were followed up every 2 months.

RNA isolation and quantitative real-time PCR. RNA was isolated from tissue samples using TRIzol (Invitrogen, Carlsbad, CA, USA), following the manufacturer's instructions. cDNA was reverse transcripted with the Fermentas reverse transcription reagents and the Applied Biosystems ${ }^{\circledR}$ TaqMan ${ }^{\circledR}$ MicroRNA Reverse Transcription kit (Applied Biosystems, Inc., Carlsbad, CA, USA). The cycling conditions were as follows: $94^{\circ} \mathrm{C}$ for $30 \mathrm{sec}, 60^{\circ} \mathrm{C}$ for $30 \mathrm{sec}$ and $72^{\circ} \mathrm{C}$ for $30 \mathrm{sec}$ for 40 cycles. The $2^{-\Delta \Delta \mathrm{Ct}}$ method was applied to analyze the data.

Cell culture and transfection. Melanoma cell line SK-Mel2 cells were maintained in RPMI-1640 medium (Invitrogen), supplemented with $10 \%$ heat-inactive fetal bovine serum (FBS; Invitrogen), $1 \%$ penicillin-streptomycin and $4 \mathrm{mmol} / 1$ L-glutamine, in a $\mathrm{CO}_{2}$ incubator at $37^{\circ} \mathrm{C}$. MicroRNA-194 mimics and negative mimics were purchased from Beijing Zoman Technology, Co., Ltd. (Beijing, China). MicroRNA-194 mimics and negative mimics were transfected into melanoma
Table I. Clinicopathological factors of the malignant melanoma patients.

\begin{tabular}{|c|c|c|c|}
\hline & Stage I/II & Stage III & Stage IV \\
\hline \multicolumn{4}{|l|}{ Gender } \\
\hline Male & 2 & 3 & 7 \\
\hline Female & 2 & 4 & 6 \\
\hline Age (years) & $62.12 \pm 5.12$ & $60.28 \pm 4.89$ & $61.45 \pm 5.04$ \\
\hline \multicolumn{4}{|l|}{ Primary site } \\
\hline Extremities & 0 & & \\
\hline Head/neck & 1 & & \\
\hline Trunk & 4 & & \\
\hline \multicolumn{4}{|l|}{ Distant sites } \\
\hline 1 & & & 0 \\
\hline 2 & & & 3 \\
\hline $3+$ & & & 10 \\
\hline \multicolumn{4}{|c|}{ Breslow thickness (mm) } \\
\hline$\leq 1.00$ & & & 0 \\
\hline $1.01-2.00$ & & & 2 \\
\hline $2.01-4.00$ & & & 4 \\
\hline$>4.00$ & & & 7 \\
\hline \multicolumn{4}{|c|}{ Lymph nodes (+) } \\
\hline 1 & & & 2 \\
\hline $2-3$ & & & 7 \\
\hline $4+$ & & & 4 \\
\hline
\end{tabular}

cell lines by using Lipofectamine 2000 (Invitrogen) according to the manufacturer's instructions.

Cell survival MTT assay. Following transfection, cells were detected using MTT (5 mg/ml, 3-(4,5-Dimethylthiazol-2-yl)2,5-diphenyltetrazolium bromide; Sigma-Aldrich, St. Louis, MO, USA) for $4 \mathrm{~h}$ at $37^{\circ} \mathrm{C}$. A total of $150 \mu \mathrm{l}$ of dimethyl sulfoxide (DMSO) assay was added into each well and optic density (OD) value was detected at $570 \mathrm{~nm}$ with a microplate reader (PerSeptive Biosystems, Inc., Framingham, MA, USA).

Annexin $V$ detection of apoptosis. Following transfection, cells were washed and resuspended in $1 \mathrm{X}$ binding buffer (BD Biosciences, Shanghai, China). Cells were incubated with Annexin V-FITC and propidium iodide (PI) $(5 \mu \mathrm{g} / \mathrm{ml}$ each) at darkness for $15 \mathrm{~min}$. Early apoptotic cells were detected using Becton-Dickinson FACSCalibur cytometer (BectonDickinson, Franklin Lakes, NJ, USA).

Caspase-3/-9 activity assay. Following transfection, cells were washed and incubated on ice in RIPA buffer. Protein concentration was detected using BCA kit (Pierce, Rockford, IL, USA). Protein lysates (100-200 $\mu \mathrm{g}$ ) were incubated with $100 \mu \mathrm{M}$ of the caspase-3 substrate DEVD-pNA (Beyotime Institute of Biotechnology, Haimen, China) or $100 \mu \mathrm{M}$ of the caspase-9 substrate Ac-LEHD-pNA (Beyotime Institute of Biotechnology) at $37^{\circ} \mathrm{C}$ for $2 \mathrm{~h}$. Optic density (OD) value was detected at $405 \mathrm{~nm}$ with a microplate reader (PerSeptive Biosystems). 


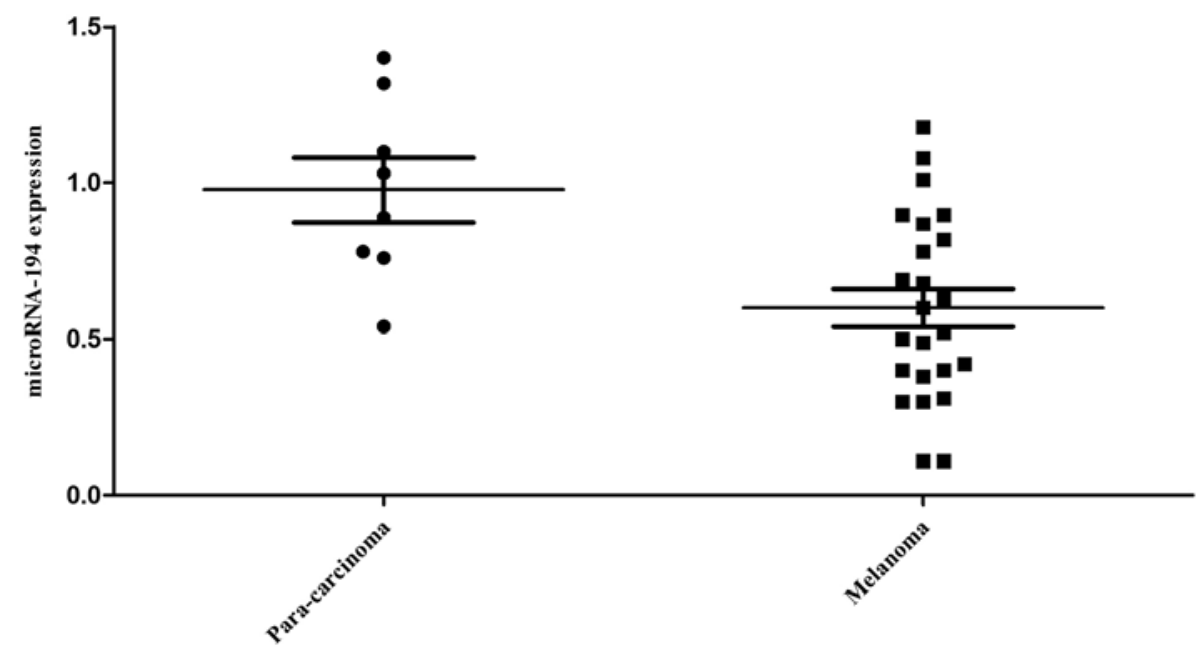

Figure 1. MicroRNA-194 expression of human melanoma patients.
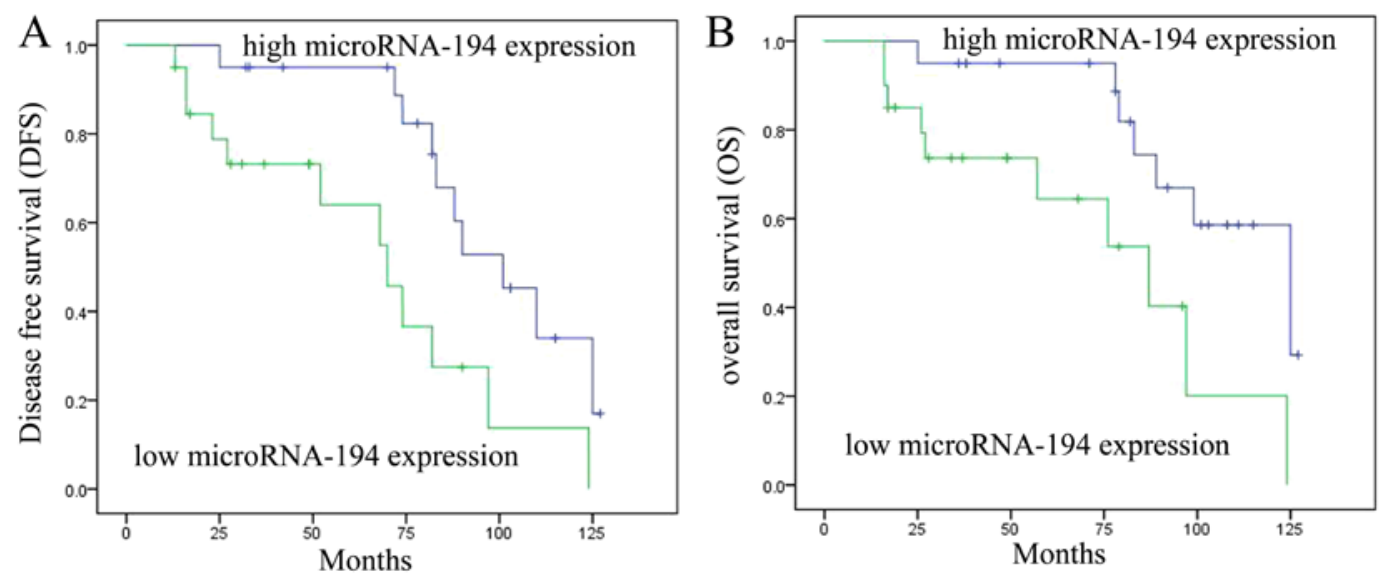

Figure 2. Disease-free survival (DFS) and overall survival (OS) of human melanoma patients. (A) DFS and (B) OS of human melanoma patients.

Western blots. Cells were washed and incubated on ice in RIPA buffer. Protein concentration was detected using BCA kit (Pierce). Protein (30-50 $\mu \mathrm{g}$ ) was separated by 6-10\% sodium dodecyl sulfate (SDS)-polyacrylamide gel electrophoresis (PAGE) and transferred onto nitrocellulose (NC) membranes (Bio-Rad Laboratories) followed by incubation with primary antibodies: Bax (1:500; Santa Cruz Biotechnology, Santa Cruz, CA, USA), Bcl-2 (1:500; Santa Cruz Biotechnology), PI3K (1:500; Santa Cruz Biotechnology), phosphorylationAKT (p-AKT, 1:500; Santa Cruz Biotechnology), FoxO3a (1:500; Santa Cruz Biotechnology), p53 (1:500; Santa Cruz Biotechnology), p21 (1:500; Santa Cruz Biotechnology) and GAPDH (1:500; Santa Cruz Biotechnology) overnight at $4^{\circ} \mathrm{C}$. Protein bands were visualized using anti-rabbit horseradish peroxidase (HRP)-conjugated secondary antibodies (1:5,000; Santa Cruz Biotechnology) and by the enhanced chemiluminescence (ECL) reagents (Bio-Rad Laboratories, Hercules, CA, USA). The bands were visualized with the Fusion FX7 system (Vilber Lourmat, Marne la Vallée, France).

Statistical analysis. All data were presented as mean \pm standard deviation (SD). The statistical significance between the groups was analyzed using Student's t-test or one-way
ANOVA. Values of $\mathrm{P}<0.05$ were considered to indicate a statistically different result.

\section{Results}

MicroRNA-194 expression of human melanoma patients. In the present study, we measured the microRNA-194 expression in MM tissues or para-carcinoma tissue using quantitative real-time PCR. As shown in Fig. 1, microRNA-194 expression of MM tissues samples was suppressed compared to that of the para-carcinoma tissue.

Disease-free survival (DFS) and overall survival (OS) of human melanoma patients. At 10 years of follow-up, we analyzed the DFS and OS of human MM patients and microRNA-194 expression. Basic information of these MM patients is shown in Table I. Fig. 2 shows that DFS and OS of high expression in human MM patients were higher than those of low expression in human MM patients.

MicroRNA-194 overexpression inhibits cell proliferation and induces apoptosis of human melanoma cells. We used microRNA-194 mimics to promote the expression of 

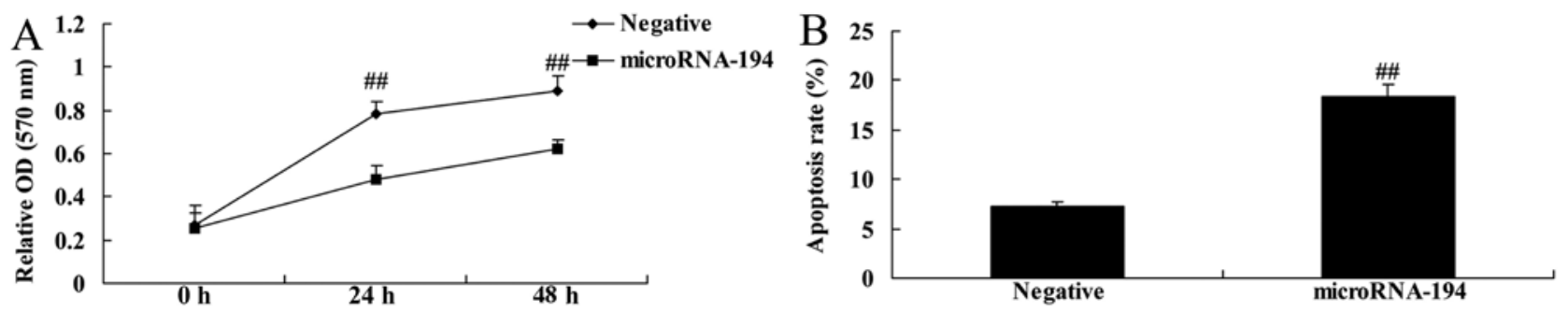

Figure 3. MicroRNA-194 overexpression inhibits cell proliferation and induces apoptosis of human melanoma cells. MicroRNA-194 overexpression inhibited cell proliferation (A) and induced apoptosis (B) of human melanoma cells. Negative, negative control group; microRNA-194, microRNA-194 overexpression group; ${ }^{\# /} \mathrm{P}<0.01$ vs. negative control group.
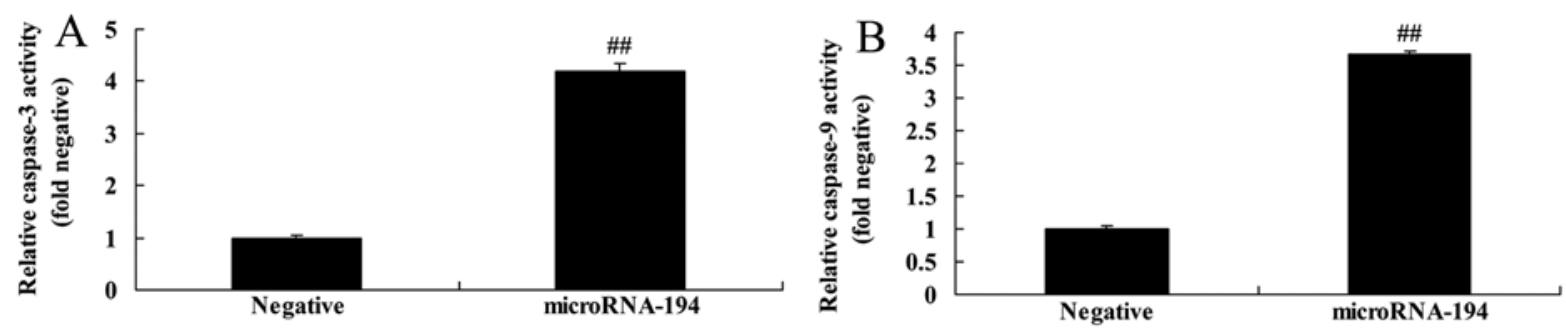

Figure 4. MicroRNA-194 overexpression increases caspase-3/-9 activities of human melanoma cells. MicroRNA-194 overexpression increased caspase-3 (A) and caspase-9 activities (B) of human melanoma cells. Negative, negative control group; microRNA-194, microRNA-194 overexpression group; ${ }^{\sharp \# ~} \mathrm{P}<0.01$ vs. negative control group.
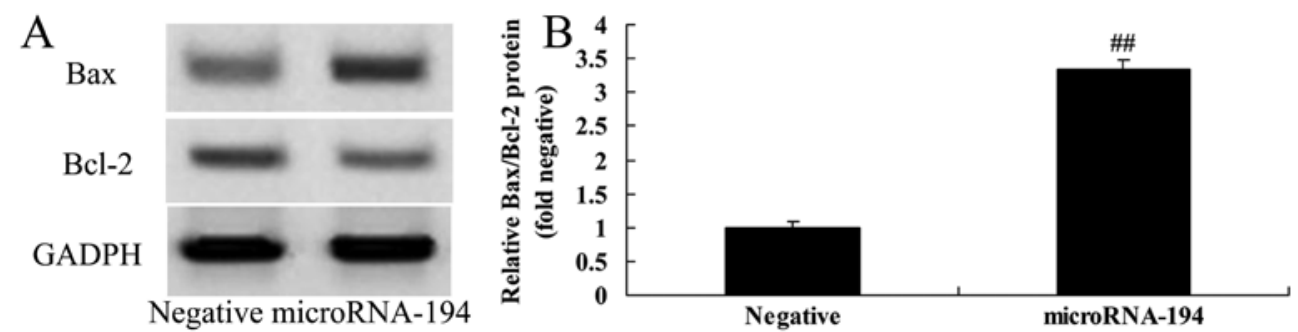

Figure 5. MicroRNA-194 overexpression promotes Bax/Bcl-2 of human melanoma cells. MicroRNA-194 overexpression promoted Bax and Bcl-2 protein expression using western blot analysis (A) and Bax and Bcl-2 protein expression using statistical analysis (B) of human melanoma cells. Negative, negative control group; microRNA-194, microRNA-194 overexpression group; ${ }^{\# \#} \mathrm{P}<0.01$ vs. negative control group.

microRNA-194 in SK-Mel2 cells and analyzed cell proliferation and apoptosis of human melanoma cells. As shown in Fig. 3, microRNA-194 overexpression inhibited cell proliferation and induced apoptosis of human melanoma cells, compared with the negative control group.

MicroRNA-194 overexpression increases caspase-3/-9 activities of human melanoma cells. We explored the function of microRNA-194 in apoptosis of human melanoma cells, and caspase-3/-9 activities were measured using commercial kits. There was a significant increase of caspase-3/-9 activities in human melanoma cells by microRNA-194 overexpression, compared with negative control group (Fig. 4).

MicroRNA-194 overexpression promotes Bax/Bcl-2 of human melanoma cells. To explore the apoptosis mechanism of microRNA-194 on human melanoma cells, Bax and Bcl-2 protein was measured using western blots. As shown in Fig. 5, microRNA-194 overexpression promoted Bax/Bcl-2 of human melanoma cells, compared with the negative control group.
MicroRNA-194 overexpression suppresses PI3K/AKT/FoxO3a signaling pathway of human melanoma cells. To determine whether PI3K/AKT/FoxO3a signaling pathway is a functional target of miR-194, we analyzed PI3K, p-AKT and FoxO3a protein expression using western blots. The results of western blots showed that PI3K, p-AKT and FoxO3a protein expression was significantly suppressed by microRNA-194 overexpression in human melanoma cells, compared with the negative control group (Fig. 6).

MicroRNA-194 overexpression induces p53/p21 signaling pathway of human melanoma cells. We determined p53/p21 signaling pathway function effect of microRNA-194 on human melanoma cells. Fig. 7 indicated that microRNA-194 overexpression significantly induced p53/p21 signaling pathway in human melanoma cells, compared with the negative control group.

Suppression of PI3K affects microRNA-194 overexpression suppressed PI3K/AKT/FoxO3a signaling pathway of human melanoma cells. Then, we employed whether the 
A

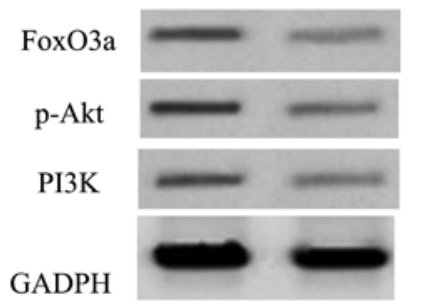

Negative microRNA-194

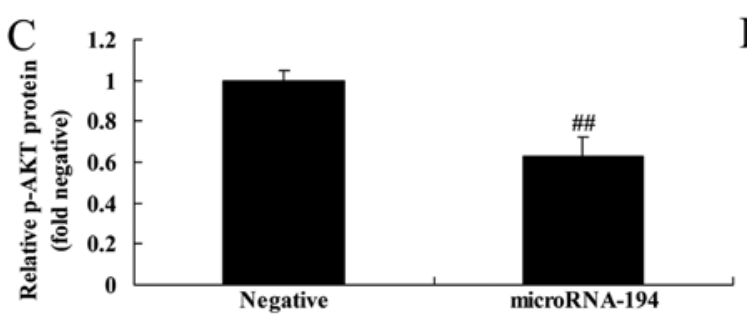

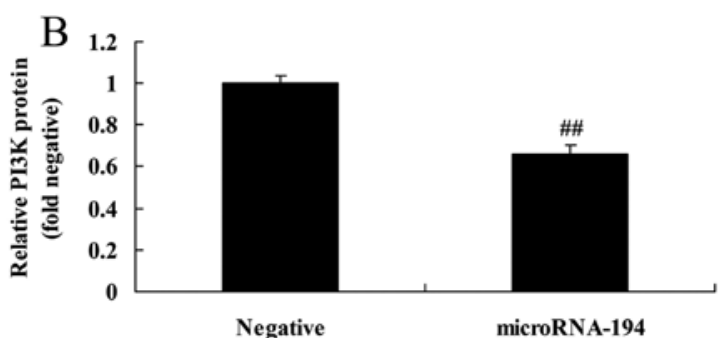

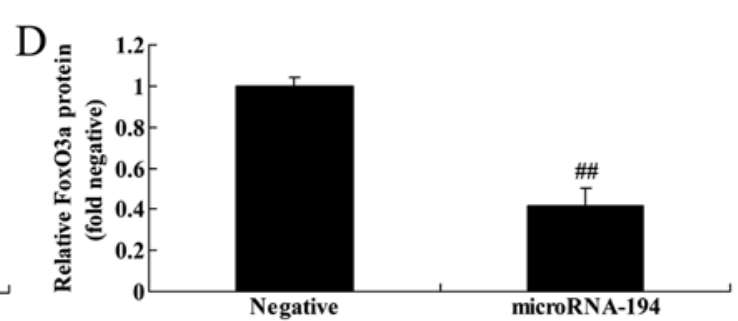

Figure 6. MicroRNA-194 overexpression suppresses PI3K/AKT/FoxO3a signaling pathway of human melanoma cells. MicroRNA-194 overexpression promoted PI3K, p-AKT and FoxO3a protein expression using western blot analysis (A) and PI3K, p-AKT and FoxO3a protein expression using statistical analysis (B-D) of human melanoma cells. Negative, negative control group; microRNA-194, microRNA-194 overexpression group; ${ }^{\# \#}$ P<0.01 vs. negative control group.
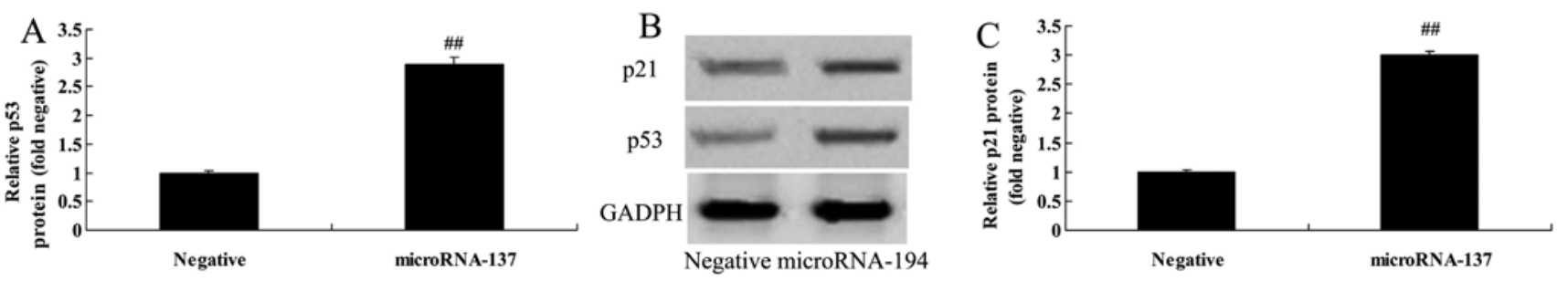

Figure 7. MicroRNA-194 overexpression induces p53/p21 signaling pathway of human melanoma cells. MicroRNA-194 overexpression promoted p53 and p21 protein expression using western blot analysis (A) and p53 and p21 protein expression using statistical analysis (B and C) of human melanoma cells. Negative, negative control group; microRNA-194, microRNA-194 overexpression group; ${ }^{\# \# ~} \mathrm{P}<0.01$ vs. negative control group.
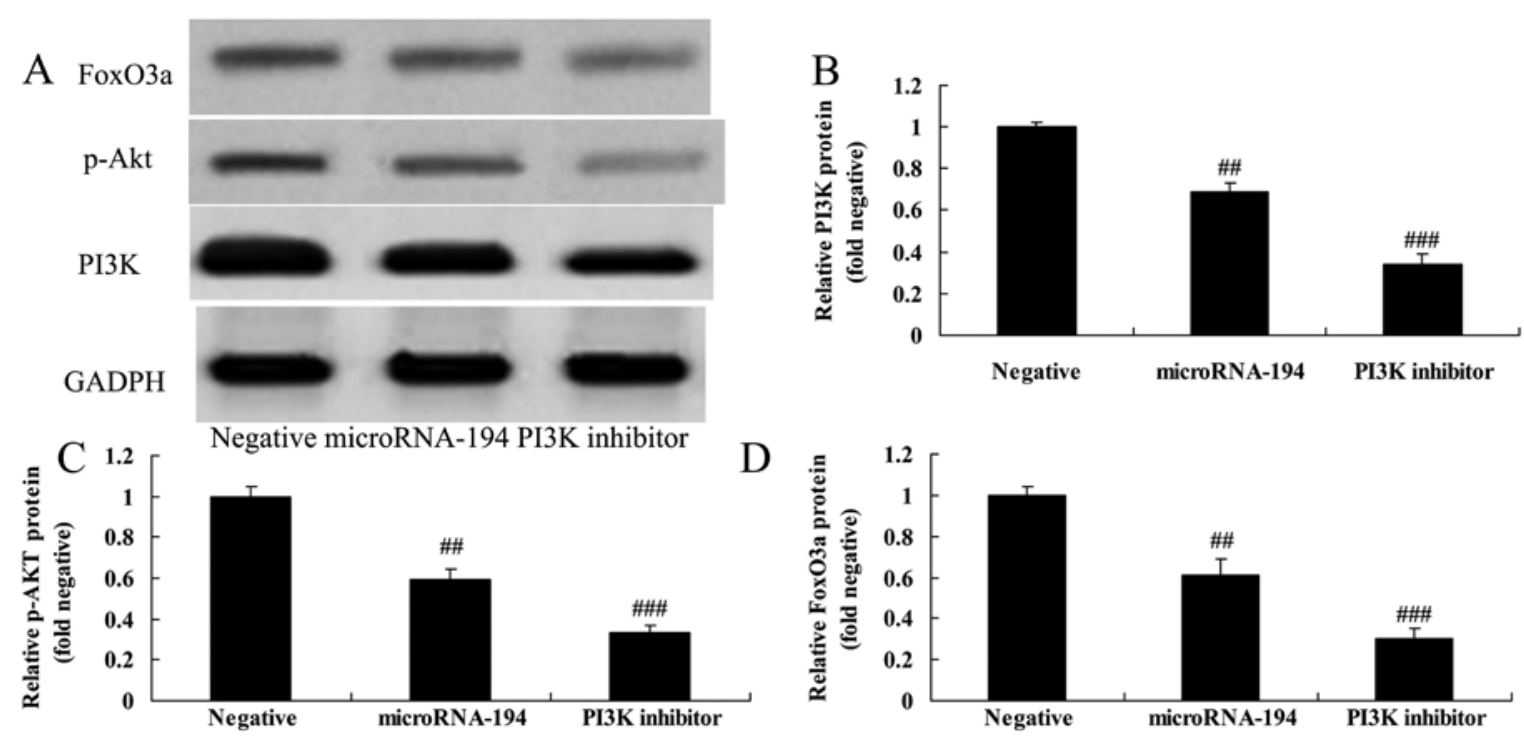

Figure 8. Suppression of PI3K affects microRNA-194 overexpression suppressed PI3K/AKT/FoxO3a signaling pathway of human melanoma cells. Suppression of PI3K affects microRNA-194 overexpression suppressed PI3K, p-AKT and FoxO3a protein expression using western blot analysis (A) and PI3K, p-AKT and FoxO3a protein expression using statistical analysis (B-D) of human melanoma cells. Negative, negative control group; microRNA-194, microRNA-194 overexpression group; PI3K inhibitor, PI3K inhibition group; ${ }^{\# \#} \mathrm{P}<0.01$ vs. negative control group, ${ }^{\# \# \#} \mathrm{P}<0.01$ vs. microRNA-194 overexpression group.

suppression of PI3K affects microRNA-194 overexpression on apoptosis of human melanoma cells. We used PI3K inhibitor, 3-Methyladenine (3-MA, 1 Mm, 48 h), significantly suppressed PI3K/AKT/FoxO3a signaling pathway in SK-Mel2 

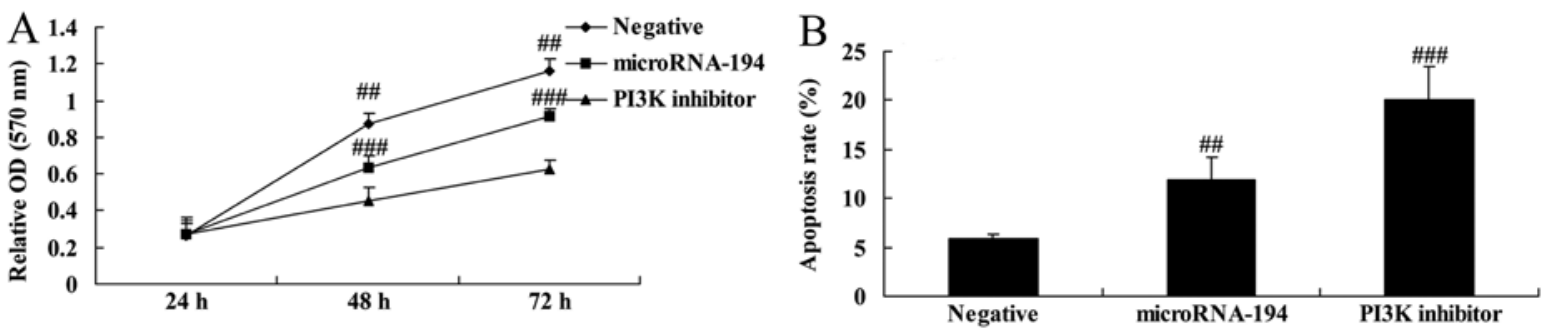

Figure 9. Suppression of PI3K affects microRNA-194 overexpression inhibited cell proliferation and induces apoptosis of human melanoma cells. Suppression of PI3K affects microRNA-194 overexpression inhibited cell proliferation (A) and induced apoptosis (B) of human melanoma cells. Negative, negative control group; microRNA-194, microRNA-194 overexpression group; PI3K inhibitor, PI3K inhibition group; ${ }^{\# \# P<0.01 ~ v s . ~ n e g a t i v e ~ c o n t r o l ~ g r o u p, ~}{ }^{\# \# \# ~} \mathrm{P}<0.01$ vs. microRNA-194 overexpression group.
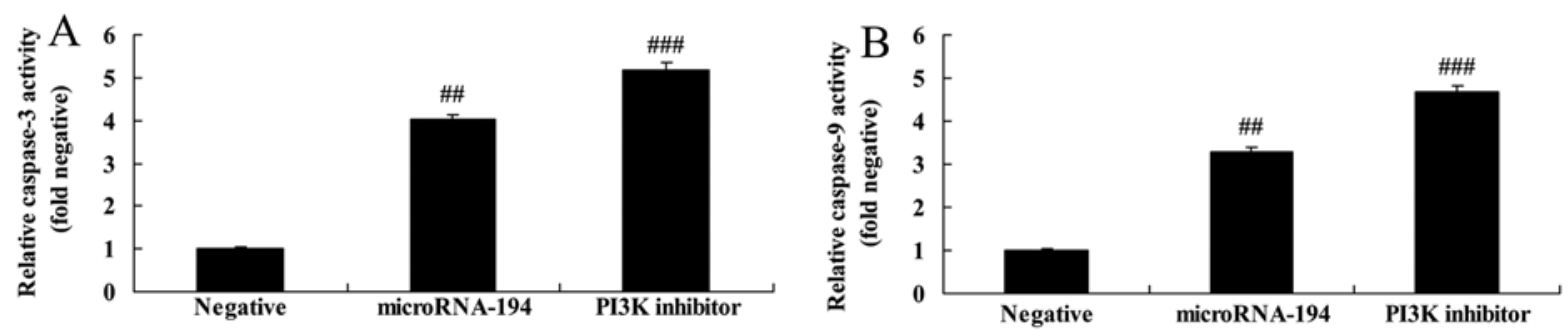

Figure 10. Suppression of PI3K affects microRNA-194 overexpression increased caspase-3/-9 activities of human melanoma cells. Suppression of PI3K affects microRNA-194 overexpression increased caspase-3 (A) and -9 activities (B) of human melanoma cells. Negative, negative control group; microRNA-194, microRNA-194 overexpression group; PI3K inhibitor, PI3K inhibition group; ${ }^{\# \#} \mathrm{P}<0.01$ vs. negative control group, ${ }^{\# \# ~} \mathrm{P}<0.01$ vs. microRNA-194 overexpression group.
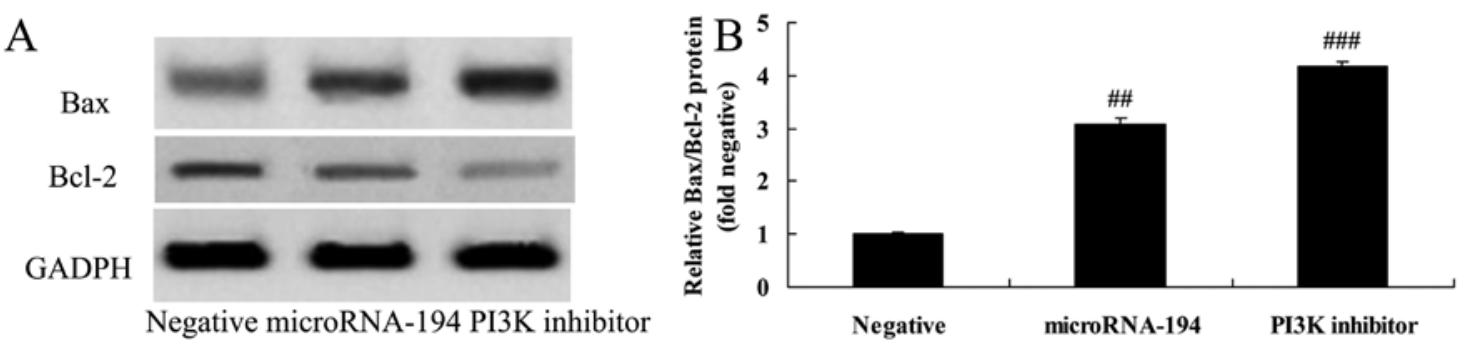

Figure 11. Suppression of PI3K affects microRNA-194 overexpression promoted Bax/Bcl-2 of human melanoma cells. Suppression of PI3K affects microRNA-194 overexpression suppressed Bax and Bcl-2 protein expression using western blot analysis (A) and Bax and Bcl-2 protein expression using statistical analysis (B) of human melanoma cells. Negative, negative control group; microRNA-194, microRNA-194 overexpression group; PI3K inhibitor, $\mathrm{PI} 3 \mathrm{~K}$ inhibition group; ${ }^{\# \#} \mathrm{P}<0.01$ vs. negative control group, ${ }^{\# \#} \mathrm{P}<0.01$ vs. microRNA-194 overexpression group.
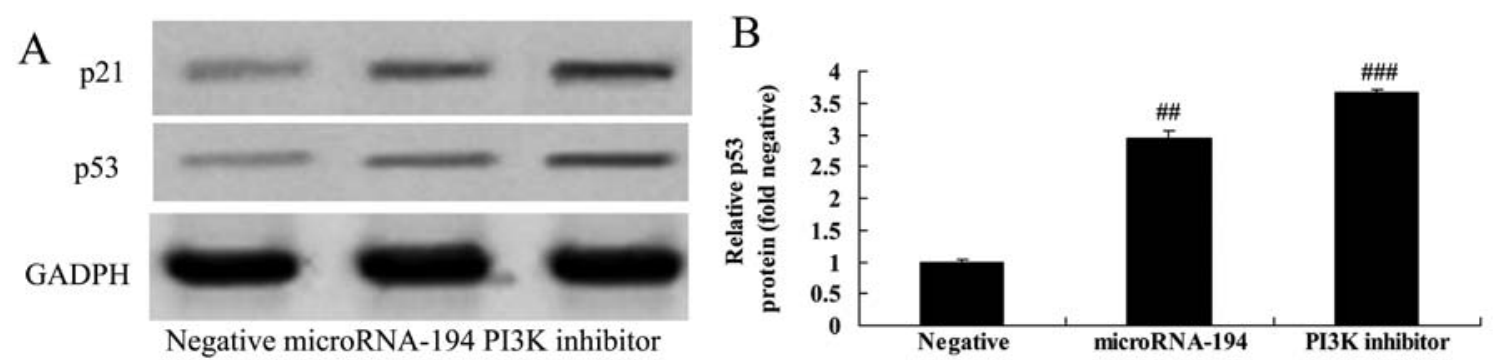

Figure 12. Suppression of PI3K affects microRNA-194 overexpression induced p53/p21 signaling pathway of human melanoma cells. Suppression of PI3K affects microRNA-194 overexpression suppressed p53 and p21 protein expression using western blot analysis (A) and p53 and p21 protein expression using statistical analysis (B and C) of human melanoma cells. Negative, negative control group; microRNA-194, microRNA-194 overexpression group; PI3K inhibitor, $\mathrm{PI} 3 \mathrm{~K}$ inhibition group; ${ }^{\# \#} \mathrm{P}<0.01$ vs. negative control group, ${ }^{\# \# \#} \mathrm{P}<0.01$ vs. microRNA-194 overexpression group.

cells following microRNA-194 overexpression, compared with the microRNA-194 overexpression group (Fig. 8). 
melanoma cells. As expected, the suppression of PI3K accelerated the anticancer effect of microRNA-194 on the cell proliferation inhibition and apoptosis induction of SK-Mel2 cells, compared with the microRNA-194 overexpression group (Fig. 9).

Suppression of PI3K affects microRNA-194 overexpression increased caspase-3/-9 activities of human melanoma cells. PI3K inhibitor significantly increased caspase-3/-9 activities in human melanoma cells by microRNA-194 overexpression, compared with microRNA-194 overexpression group (Fig. 10).

Suppression of PI3K affects microRNA-194 overexpression promoted Bax/Bcl-2 of human melanoma cells. We determined whether PI3K inhibition affects microRNA-194 overexpression promoted $\mathrm{Bax} / \mathrm{Bcl}-2$ of human melanoma cells. As shown in Fig. 11, PI3K inhibition significantly increased Bax/Bcl-2 of human melanoma cells following microRNA-194 overexpression, compared with the microRNA-194 overexpression group (Fig. 11).

Suppression of PI3K affects microRNA-194 overexpression induced p53/p21 signaling pathway of human melanoma cells. We assess whether PI3K inhibition affects p53/p21 signaling pathway of human melanoma cells following microRNA-194 overexpression. Compared with microRNA-194 overexpression, PI3K inhibition significantly induced p53/p21 signaling pathway of human melanoma cells following microRNA-194 overexpression (Fig. 12).

\section{Discussion}

Malignant melanoma (MM), is derived from the melanocytes in the neural crest, and it is the most fatal skin tumor (17). It is associated with extremely high grade of malignancy and hidden onset, and the majority already has metastases when discovered in clinic, thus, it has poor chemoradiotherapy effects and great treatment difficulties (18). MM patients account for $80 \%$ among the patients who die of skin cancer (19). In recent years, the morbidity and mortality of MM show an increasing trend year by year, which greatly threaten human health (19). Our results found that microRNA-194 expression of MM tissues samples was suppressed compared to that of para-carcinoma tissue, and DFS and OS of high expression in MM patients were higher than those of low expression in MM patients.

Akt is also called protein kinase B, which is a kind of serine/threonine protein kinase. As previously reported, the PI3K pathway in a variety of malignancies is active, and the downstream signaling molecules of PI3K (Akt, mTOR, PDK and ILK) are the potential targets for treating malignancies $(20,21)$. Human Foxo transcription factor family (forkhead box protein family) has four members, the Foxo1, Foxo2, Foxo3a and Foxo4, which participate in regulating multiple cellular processes, including cell proliferation, apoptosis, differentiation and metabolism (22). Foxo family is an important downstream signaling molecule of the PI3K/Akt signal pathway (22). Foxo family transfers from the nucleus to the cytoplasm after being phosphorylated by Akt and its transcriptional activity is inhibited (23). We demonstrated that microRNA-194 overexpression inhibited cell proliferation, induced apoptosis, increased caspase-3/-9 activities and promoted $\mathrm{Bax} / \mathrm{Bcl}-2$ of human melanoma cells through suppression of PI3K/AKT/FoxO3a signaling pathway.

Foxo has the function of inhibiting the intracellular reactive oxygen, which is mainly through promoting the expression of reactive oxygen scavenger (23). The reactive oxygen scavengers include superoxide dismutase (SOD2, MnSOD) and catalase. Akt has long been considered as the signal pathway for promoting tumor survival and proliferation, besides, in multiple tumor cells, Akt is in the excessively activated state (24). However, it has also been considered that the excessively activated Akt could inhibit cell apoptosis due to numerous reasons, except for the reactive oxygen-induced apoptosis; furthermore, the activation of Akt made the cells more sensitive to the reactive oxygen-induced apoptosis (25). Altogether, these data indicated that PI3K inhibition significantly suppressed PI3K/AKT/FoxO3a signaling pathway in SK-Mel2 cells following microRNA-194 overexpression. Chi (26) provided compelling evidence that microRNA-194 inhibited cell proliferation of oral squamous cell carcinoma by reducing PI3K-Akt-FoxO3a signaling. Our results were consistent with previous studies demonstrating that microRNA-194 inhibits biological features of human MM through PI3K/AKT/ FoxO3a signaling pathway. Krützfeldt et al (27) provided compelling evidence that microRNA-194 regulates cell proliferation of oral squamous cell carcinoma via PI3K/AKT/ FoxO3a signaling pathway.

The transcription of p53, as well as mRNA splicing and translation is regulated, the mRNA splicing prompts the p53 gene to express several isomeric molecules which have various activities (28). p53 has extremely short half-life in normal cells, which is only 5-30 min. The intracellular p53 level is mainly regulated by the rigid negative feedback of its target gene murine double minute 2 (MDM2), and its transcript MDM2 is a kind of E3 ubiquitin ligase, which can promote its degradation through promoting the ubiquitination of p53 (29). However, MDM2 has the function of regulating the ubiquitin-independent p53 activity, and it can prompt p53 to adopt the mutant conformation through binding its acidic domain with $\mathrm{p} 53$, and thus inhibit the DNA binding activity of p53 (30). Furthermore, MDM2 is a target gene of p53 activation; therefore, p53-MDM2 forms a negative feedback circuit, which means that p53 regulates the expression of MDM2 at the transcription level, while MDM2 regulates the transcriptional activity of p53 as well as the degradation of the proteasome pathway that it mediates (29). In the present study, we demonstrated that microRNA-194 overexpression induced p53/p21 signaling pathway of human melanoma cells.

p21 is an apoptosis regulatory factor, the activated AKT1 can phosphorylate the Thr145 residue of p21, and thus block the nuclear translocation of $\mathrm{p} 21$, render the arrest of $\mathrm{p} 21$ in the cytoplasm; and the phosphorylated p21 which locates in cytoplasm has anti-apoptotic activity, which is due to inhibition of the activity of proteins that are involved in apoptosis, such as procaspase- 3 , caspase- 8 and caspase-10; in addition, it can also upregulate the expression of the cytokines that have anti-apoptotic activity, and inhibit the expression of the pro-apoptotic genes transcribed by MYC and E2F1 $(31,32)$.The transcription of p21 is regulated by multiple signals and factors. Under the 
induction of various stimulations, such as DNA damage and nucleotide loss, the transcription of p21 depends on p53 as well as its family member p73 (33). Furthermore, many other factors can directly or indirectly induce p53-independent p21 transcription (34).

It is currently considered that the p53-p21 pathway can induce aging, while the p16-pRB pathway maintains aging. In-depth research reveals that p53 induces aging through its downstream p21 and ROS; to be more specific, certain level and duration of ROS is the determinant of p53 initiating the p21 transcription, while the increased p21 expression can exert its transcription regulatory function through the interaction with the transcriptional coactivator $\mathrm{p} 300$, which does not rely on PCNA binding or CDK kinase inhibitory activity; induce PIG3 expression and thus induce elevated ROS in cells; and the elevated ROS can maintain long-term cell cycle arrest $(14,35)$. If ROS is eliminated from the p21-induced aging cells, the cells can recover from aging (14). Though p16 is also CDK inhibitory factor, it does not have transcription regulatory function and cannot induce elevated ROS level in the cell; therefore, it can induce irreversible cell cycle arrest (36). In the present study, our in vitro studies showed that PI3K inhibition significantly induced p53/p2 signaling pathway in SK-Mel2 cells following microRNA-194 overexpression. Sundaram et al (37) reported that miR-194 inhibits thrombospondin-1 and promotes angiogenesis of colon cancers through p53 signaling pathway. Taken together, these data provide solid evidence to support that microRNA-194 exerts it inhibitory effect on human melanoma cells, at least in part, through p53/p21 signaling pathway. Krützfeldt et al (27) found that miR-194 is a target of transcription factor 1 and regulates Dgcr8 and p53 in liver tumorigenesis.

In conclusion, our results indicate that microRNA-194 overexpression inhibited cell proliferation, induced apoptosis, increased caspase-3/-9 activities and promoted Bax/Bcl-2 of human melanoma cells via PI3K/AKT/FoxO3a and p53/p21 signaling pathway. Therefore, the microRNA-194/PI3K/AKT/ FoxO3a and p53/p21 should be further studied for possible treatment of melanoma.

\section{References}

1. Greene JM, Schneble EJ, Jackson DO, Hale DF, Vreeland TJ, Flores M, Martin J, Herbert GS, Hardin MO, Yu X, et al: A phase I/IIa clinical trial in stage IV melanoma of an autologous tumor-dendritic cell fusion (dendritoma) vaccine with low dose interleukin-2. Cancer Immunol Immunother 65: 383-392, 2016.

2. Ong CC, Jubb AM, Jakubiak D, Zhou W, Rudolph J, Haverty PM, Kowanetz M, Yan Y, Tremayne J, Lisle R, et al: P21-activated kinase 1 (PAK1) as a therapeutic target in BRAF wild-type melanoma. J Natl Cancer Inst 105: 606-607, 2013.

3. Benyi E, Kieler H, Linder M, Ritzén M, Carlstedt-Duke J, Tuvemo T, Westphal O and Sävendahl L: Risks of malignant and non-malignant tumours in tall women treated with high-dose oestrogen during adolescence. Horm Res Paediatr 82: 89-96, 2014.

4. Eggermont AM, Suciu S, Rutkowski P, Kruit WH, Punt CJ, Dummer R, Salès F, Keilholz U, de Schaetzen G and Testori A; EORTC Melanoma Group: Long term follow up of the EORTC 18952 trial of adjuvant therapy in resected stage IIB-III cutaneous melanoma patients comparing intermediate doses of interferon-alpha-2b (IFN) with observation: Ulceration of primary is key determinant for IFN-sensitivity. Eur J Cancer 55: 111-121, 2016.
5. Varamo C, Occelli M, Vivenza D, Merlano M and Lo Nigro C: MicroRNAs role as potential biomarkers and key regulators in melanoma. Genes Chromosomes Cancer 56: 3-10, 2016.

6. Babapoor S, Horwich M, Wu R, Levinson S, Gandhi M, Makkar H, Kristjansson A, Chang M and Dadras SS: microRNA in situ hybridization for miR-211 detection as an ancillary test in melanoma diagnosis. Mod Pathol 29: 461-475, 2016.

7. Venkatesan N, Kanwar J, Deepa PR, Khetan V, Crowley TM, Raguraman R, Sugneswari G, Rishi P, Natarajan V, Biswas J, et al: Clinico-pathological association of delineated miRNAs in uveal melanoma with monosomy $3 /$ disomy 3 chromosomal aberrations. PLoS One 11: e0146128, 2016.

8. Dror S, Sander L, Schwartz H, Sheinboim D, Barzilai A, Dishon Y, Apcher S, Golan T, Greenberger S, Barshack I, et al: Melanoma miRNA trafficking controls tumour primary niche formation. Nat Cell Biol 18: 1006-1017, 2016.

9. Kou Y, Li L, Li H, Tan Y, Li B, Wang K and Du B: Berberine suppressed epithelial mesenchymal transition through cross-talk regulation of PI3K/AKT and RAR $\alpha / \mathrm{RAR} \beta$ in melanoma cells. Biochem Biophys Res Commun 479: 290-296, 2016.

10. Xu G, Zhang W, Bertram P, Zheng XF and McLeod H: Pharmacogenomic profiling of the PI3K/PTEN-AKT-mTOR pathway in common human tumors. Int J Oncol 24: 893-900, 2004.

11. Dong MH, Zhang Q, Wang YY, Zhou BS, Sun YF and Fu Q: Euphorbia fischeriana Steud inhibits malignant melanoma via modulation of the phosphoinositide-3-kinase/Akt signaling pathway. Exp Ther Med 11: 1475-1480, 2016.

12. Doscas ME, Williamson AJ, Usha L, Bogachkov Y, Rao GS, Xiao F, Wang Y, Ruby C, Kaufman H, Zhou J, et al: Inhibition of p70 S6 kinase (S6K1) activity by A77 1726 and its effect on cell proliferation and cell cycle progress. Neoplasia 16: 824-834, 2014.

13. Guo Y, Chang H, Li J, Xu XY, Shen L, Yu ZB and Liu WC: Thymosin alpha 1 suppresses proliferation and induces apoptosis in breast cancer cells through PTEN-mediated inhibition of PI3K/ Akt/mTOR signaling pathway. Apoptosis 20: 1109-1121, 2015.

14. Guterres FA, Martinez GR, Rocha ME and Winnischofer SM: Simvastatin rises reactive oxygen species levels and induces senescence in human melanoma cells by activation of p53/p21 pathway. Exp Cell Res 319: 2977-2988, 2013.

15. de Andrade BA, León JE, Carlos R, Delgado-Azañero W, Mosqueda-Taylor A and de Almeida OP: Immunohistochemical expression of p16, p21, p27 and cyclin D1 in oral nevi and melanoma. Head Neck Pathol 6: 297-304, 2012.

16. Jang GH and Lee M: BH3-mimetic gossypol-induced autophagic cell death in mutant BRAF melanoma cells with high expression of p21Cip ${ }^{1}$ ). Life Sci 102: 41-48, 2014.

17. van der Ploeg AP, Haydu LE, Spillane AJ, Scolyer RA, Quinn MJ, Saw RP, Shannon KF, Stretch JR and Thompson JF: Melanoma patients with an unknown primary tumor site have a better outcome than those with a known primary following therapeutic lymph node dissection for macroscopic (clinically palpable) nodal disease. Ann Surg Oncol 21: 3108-3116, 2014.

18. Plimack ER, Desai JR, Issa JP, Jelinek J, Sharma P, Vence LM, Bassett RL, Ilagan JL, Papadopoulos NE and Hwu WJ: A phase I study of decitabine with pegylated interferon $\alpha-2 b$ in advanced melanoma: Impact on DNA methylation and lymphocyte populations. Invest New Drugs 32: 969-975, 2014.

19. Robert C, Dummer R, Gutzmer R, Lorigan P, Kim KB, Nyakas M, Arance A, Liszkay G, Schadendorf D, Cantarini M, et al: Selumetinib plus dacarbazine versus placebo plus dacarbazine as first-line treatment for BRAF-mutant metastatic melanoma: A phase 2 double-blind randomised study. Lancet Oncol 14: 733-740, 2013.

20. Wang K, Gao W, Dou Q, Li Q, Nice EC and Huang C: Ivermectin induces PAK1-mediated cytostatic autophagy in breast cancer. Autophagy 12: 2498-2499, 2016.

21. Vucicevic L, Misirkic M, Janjetovic K, Vilimanovich U, Sudar E, Isenovic E, Prica M, Harhaji-Trajkovic L, Kravic-Stevovic T, Bumbasirevic V,et al: Compound $\mathrm{C}$ induces protective autophagy in cancer cells through AMPK inhibition-independent blockade of Akt/mTOR pathway. Autophagy 7: 40-50, 2011.

22. Syed DN, Chamcheu JC, Khan MI, Sechi M, Lall RK, Adhami VM and Mukhtar H: Fisetin inhibits human melanoma cell growth through direct binding to p70S6K and mTOR: Findings from 3-D melanoma skin equivalents and computational modeling. Biochem Pharmacol 89: 349-360, 2014. 
23. Babchia N, Calipel A, Mouriaux F, Faussat AM and Mascarelli F: The PI3K/Akt and mTOR/P70S6K signaling pathways in human uveal melanoma cells: Interaction with B-Raf/ERK. Invest Ophthalmol Vis Sci 51: 421-429, 2010.

24. Caporali S, Alvino E, Lacal PM, Levati L, Giurato G, Memoli D, Caprini E, Antonini Cappellini GC and D'Atri S: Targeting the $\mathrm{PI} 3 \mathrm{~K} / \mathrm{AKT} / \mathrm{mTOR}$ pathway overcomes the stimulating effect of dabrafenib on the invasive behavior of melanoma cells with acquired resistance to the BRAF inhibitor. Int J Oncol 49: 1164-1174, 2016

25. Pappalardo F, Russo G, Candido S, Pennisi M, Cavalieri S, Motta S, McCubrey JA, Nicoletti F and Libra M: Computational modeling of PI3K/AKT and MAPK signaling pathways in melanoma cancer. PLoS One 11: e0152104, 2016.

26. Chi H: miR-194 regulated AGK and inhibited cell proliferation of oral squamous cell carcinoma by reducing PI3K-Akt-FoxO3a signaling. Biomed Pharmacother 71: 53-57, 2015.

27. Krützfeldt J, Rösch N, Hausser J, Manoharan M, Zavolan M and Stoffel M: MicroRNA-194 is a target of transcription factor 1 (Tcf1, HNF1 $\alpha$ ) in adult liver and controls expression of frizzled-6. Hepatology 55: 98-107, 2012.

28. Lawrence NF, Hammond MR, Frederick DT, Su Y, DiasSantagata D, Deng A, Selim MA, Mahalingam M, Flaherty KT and Hoang MP: Ki-67, p53, and p16 expression, and G691S RET polymorphism in desmoplastic melanoma (DM): A clinicopathologic analysis of predictors of outcome. J Am Acad Dermatol 75: 595-602, 2016.

29. Perrotta C, Buonanno F, Zecchini S, Giavazzi A, Proietti Serafini F, Catalani E, Guerra L, Belardinelli MC, Picchietti S, Fausto AM, et al: Climacostol reduces tumour progression in a mouse model of melanoma via the p53-dependent intrinsic apoptotic programme. Sci Rep 6: 27281, 2016.

30. Zhao S, Niu F, Xu CY, Ye L, Bi GB, Chen L, Gong P, Tian G and Nie TH: Microarray and ChIP-seq data analysis revealed changes in $\mathrm{p} 53$-mediated transcriptional regulation in Nutlin-3treated U2OS cells. Mol Med Rep 12: 4284-4290, 2015.
31. Kaluzki I, Hrgovic I, Hailemariam-Jahn T, Doll M, Kleemann J, Valesky EM, Kippenberger S, Kaufmann R, Zoeller N and Meissner M: Dimethylfumarate inhibits melanoma cell proliferation via p21 and p53 induction and bcl-2 and cyclin B1 downregulation. Tumour Biol 37: 13627-13635, 2016.

32. Fan T, Jiang S, Chung N, Alikhan A, Ni C, Lee CC and Hornyak TJ: EZH2-dependent suppression of a cellular senescence phenotype in melanoma cells by inhibition of $\mathrm{p} 21 /$ CDKN1A expression. Mol Cancer Res 9: 418-429, 2011.

33. Genov M, Kreiseder B, Nagl M, Drucker E, Wiederstein M, Muellauer B, Krebs J, Grohmann T, Pretsch D, Baumann K, et al: Tetrahydroanthraquinone derivative $( \pm)$-4-deoxyaustrocortilutein induces cell cycle arrest and apoptosis in melanoma cells via upregulation of p21 and p53 and downregulation of NF-kappaB. J Cancer 7: 555-568, 2016.

34. Yun CY, You ST, Kim JH, Chung JH, Han SB, Shin EY and Kim EG: p21-activated kinase 4 critically regulates melanogenesis via activation of the CREB/MITF and $\beta$-catenin/MITF pathways. J Invest Dermatol 135: 1385-1394, 2015.

35. Pelletier J, Dayan F, Durivault J, Ilc K, Pécou E, Pouysségur J and Mazure NM: The asparaginyl hydroxylase factor-inhibiting HIF is essential for tumor growth through suppression of the p53-p21 axis. Oncogene 31: 2989-3001, 2012.

36. Nicolau-Galmés F, Asumendi A, Alonso-Tejerina E, PérezYarza G, Jangi SM, Gardeazabal J, Arroyo-Berdugo Y, Careaga JM, Díaz-Ramón JL, Apraiz A, et al: Terfenadine induces apoptosis and autophagy in melanoma cells through ROS-dependent and -independent mechanisms. Apoptosis 16: 1253-1267, 2011.

37. Sundaram P, Hultine S, Smith LM, Dews M, Fox JL, Biyashev D, Schelter JM, Huang Q, Cleary MA, Volpert OV, et al: p53-responsive miR-194 inhibits thrombospondin-1 and promotes angiogenesis in colon cancers. Cancer Res 71: 7490-7501, 2011. 